\title{
Trichomonas Prostatitis
}

National Cancer Institute

\section{Source}

National Cancer Institute. Trichomonas Prostatitis. NCI Thesaurus. Code C35176.

Infection of the prostate gland caused by Trichomonas vaginalis. 